\title{
Untapped potential: Mining Register of Chinese Immigrants to Canada, 1886-1949 using R and Palladio
}

\author{
Sarah Zhang \\ Simon Fraser University \\ s_zhang@sfu.ca \\ Allan Cho \\ The University of British Columbia \\ allan.cho@ubc.ca
}

\begin{abstract}
Register of Chinese Immigrants to Canada, 1886-1949 is a historical dataset openly available to the public. Drawing on the previous studies based on the data, this study aims to lower the barriers to the data use, as well as to reveal substantive potential of the data that has not been leveraged by scholars.
\end{abstract}

Keywords: Digital Humanities, Chinese immigrants, headtax, Canada, migration pattern, $R$, network analysis 


\section{Introduction}

Between 1885 and 1923, the Canadian government imposed a head tax on Chinese immigrants entering Canada in order to restrict immigration. While a print register was created to keep track of the influx of migrants, these detailed recordings have actually provided researchers and historians with years of demographic information about the immigrants and have become a rich source of data for researchers. Painstakingly transformed to a Microsoft Excel spreadsheet, a project led by historians at the University of British Columbia (UBC), these researchers have provided original records that include 97,123 registrants whose arrival time in Canada had spread out over half a century from 1886 to $1949^{1}$. Though an impressively large-scale dataset, these records are incoherent as they show idiosyncratic dialects of the immigrants which result in variations of place names and titles. The inconsistencies in place names, unfortunately, lead to difficulties for anyone who wishes to exercise any analysis associated with the immigrants' origins. In other words, while there is a treasure trove of data to use, it may be unusable for most unless there can be data manipulation that can unlock a better understanding of the missing gaps.

To address these inconsistencies, the UBC Asian Library initiated a project to normalize various transliterations of the immigrants' origins laid the groundwork for more in-depth research for future researchers. The immigrants' origins are represented at two hierarchical levels: county and villages/towns; there are eight counties and numerous villages in the registry. Of the eight counties, the names of villages/towns in three counties have been mapped: Sun Woy (now known as Xinhui), Zhongshan, and Taishan. Although just a snippet of the records, this normalized data offers a true glimpse into the full impact of what is available in the research.

\footnotetext{
${ }^{1}$ The dataset is openly accessible at the Open Collections of UBC: https://open.library.ubc.ca/clRcle/collections/facultyresearchandpublications/52383/items/1.0075988
} 
Since the completion of the digitization work, scholarship has drawn on the digital records from the project, manifesting differing methods and research findings. Peter Ward's publication in 2013 focused on the changes on the wellbeing of Chinese headtax immigrants, particularly analyzing the immigrants' stature, a statistical indicator for wellbeing. He contrasted mean height by age of different age cohorts (10 years apart), and found a rising trend in stature over time: "a slow but significant increase in stature within the immigrant population from the middle of the 19th century to the early years of the Sino-Japanese War. "(p. 494) This increase in height, Ward speculated, can be attributed to the migration process itself.

Subsequent to Ward's study, other researchers continued pursuing historical inquiries using digital humanities tools, specifically GIS(Geographic Information System) to investigate the spatial distribution of migration. Hermansen and Yu (2014) studies the spatial elements in the headtax registry and visualized the distribution of the Canadian destinations of Chinese immigrants. This first attempt at examining the spatial distribution of the immigrants' destinations in Canada, however, only depicted a big picture rather than zooming in on nuanced patterns of migration such as kinship networks. As a further step, Yu and Chen (2017) used Gephi, a network analysis tool, to reveal patterns of family chain migration, and found that "the four major Cantonese clans in Saskatchewan all showed a high geographic distribution in the choice of destinations". (p.46)

Looking back at what has been done up to this point -- the digitization of the original registry, normalization of origins, and scholarly studies that have arisen from the digital records -- the authors, both academic librarians, became curious about furthering the research into the historical data that has been used. They were specifically interested in whether the normalized origins have 
been leveraged, and whether the findings of the previous studies can represent a coherent picture.

In terms of methodology, the authors, feeling the previous studies discussed above haven't demonstrated the use of a great variety of computational tools, employed $\mathrm{R}$, a statistical computational language, and Palladio, a network analysis tool developed by the Humanities + Design Lab at Stanford University.

The intentions of this study are therefore twofold: first, the authors hope to demonstrate the untapped potential in the head tax data. Second, this study will provide testimony for new modes that librarians help shape digital scholarship and create promising new research questions for researchers.

\section{Analyzing the immigrants' wellbeing at the level of county}

\section{Method}

The normalization of origins project has opened up the possibility of extracting nuances information of the immigrants who came from the same county and same village. Hoping to test whether Ward's conclusions about the rising height of immigrants over time is true at one finer level--county, similar research methodology to Ward's study was employed and analyzed the stature of the immigrants from Xinhui county.

\section{Findings}

Figure 1: Mean Height by age (male and female) $n=45,983(m), 810(f)$

Source: Ward, 2013 


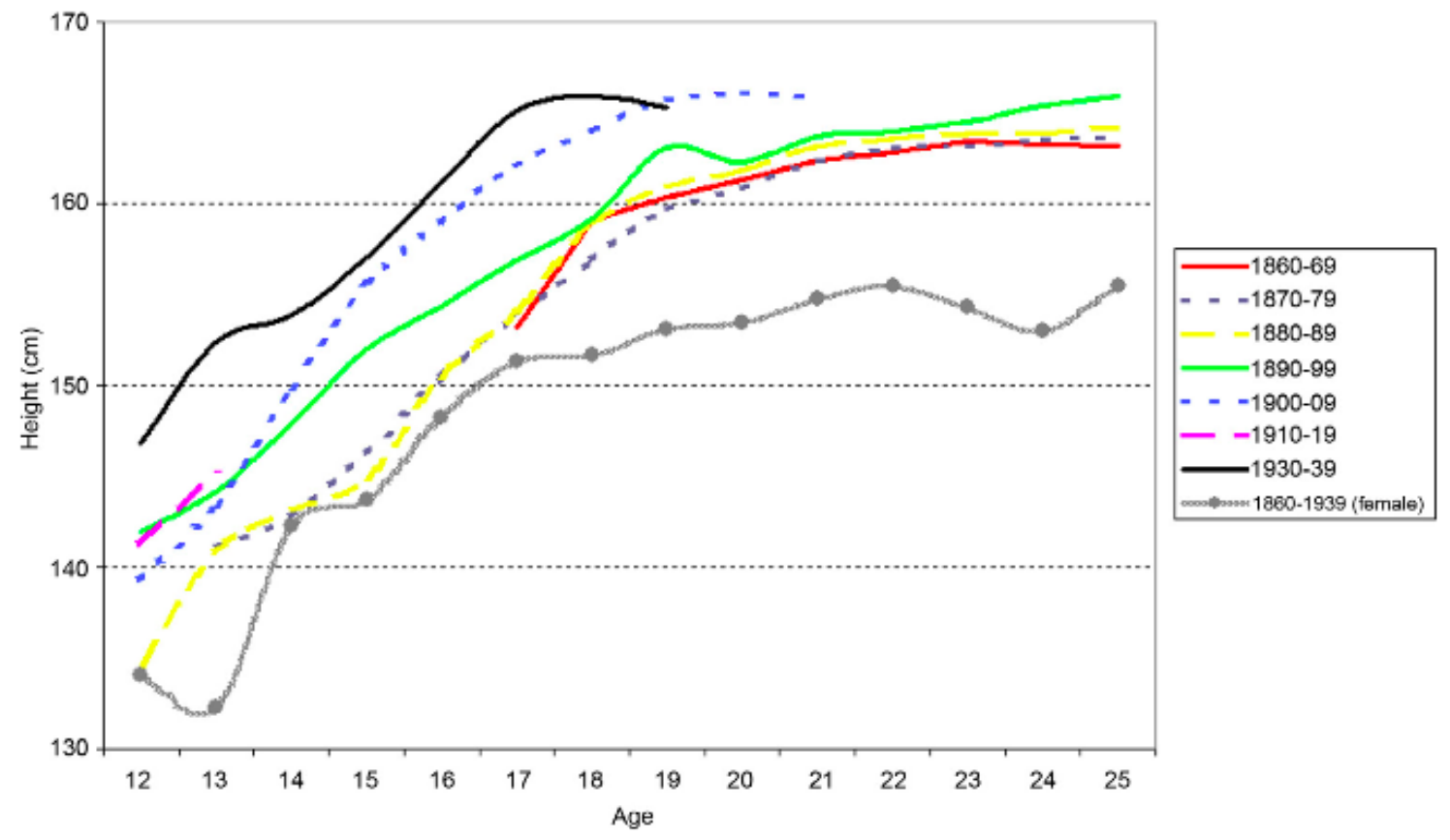

Figure 2: Mean Height by Age, contrasting 5 birth year cohort. $(n=11,839)$ Immigrants from Xinhui 


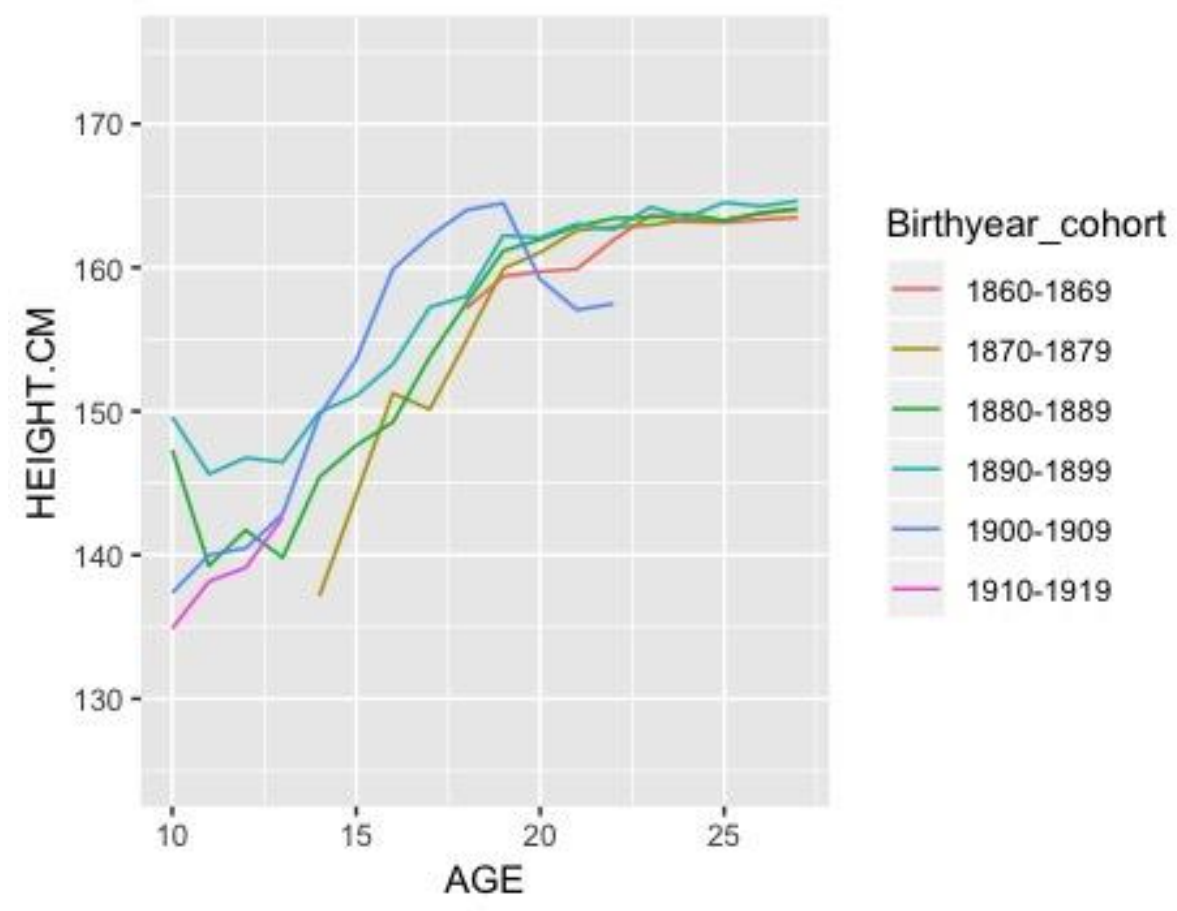

This graph reveals a very different picture of height change over the age among the different birth cohorts from Ward's graph. Specifically the patterns emerging from it include:

1) In Ward's graph, there is a direct correlation with birth cohort. For example, the line of 1930-1939 holds the highest place on the place. Sequentially, from highest to lowest line: 1930-39>1900-09>1890-99>1879-79>1860-69, meaning the mean height of the cohorts in later years is higher than that of in earlier years. For this Xinhui graph, this is not true. Only three cohorts mostly follow this pattern: 1890-99, 1880-89, and 1870-79.

2) For 5 out of 6 cohorts, the age-height relation seems to be linear or near-linear. But the 1900-1909 cohort (the yellow line) doesn't fit in well with this pattern, manifesting a highly tumbling line. Compared to other lines, this cohort presents a much steeper growth during 
the adolescent years (age 14 to 19), followed by a plummet during the age 19 to 22 . In other words, there were dramatic changes in heights for the 1900-1909 cohort.

\section{Discussions}

In analyzing all the Chinese immigrants' registries, Ward's study discovered a rising trend in stature over time, which he speculated to be tied to the migration process itself. He hypothesized that the financial remittances from the migrants back to China had a direct correlation to the increase in stature of migrants of subsequent generations who benefitted from the dietary improvements. Additionally, he found there was adolescent spurt for almost all the cohorts (Ward, 2013).

And yet, when focused exclusively on immigrants from Xinhui county, the findings do not fit neatly with Ward's narrative. Instead, very different patterns and nuanced trends in the immigrants' heights are uncovered. For example, the birth year cohort of 1900-1909 and 1910-1919 present surprising and unexpected trends for immigrants originated from Xinhui: why is the mean height for most ages between 1910 and 1919 cohort the lowest, instead of highest, among all the cohorts? Why did the mean height for adolescent years grow so quickly, while plummeting in following age group, for the 1900-09 cohort? Answering these questions would be meaningful work, because height is an indicator for the immigrants' wellbeing before they landed in Canada. Our intention, however, is not to try to answer these questions by ourselves but simply to expose these patterns which could serve as gateways to more in-depth historical studies on the immigrants from Guangdong in the late nineteenth century and early twentieth century. 


\section{Analyzing migration patterns at the level of village}

\section{Method}

According to the assumption that migrants who share a surname and who came from the same village are "family" from the same clan," as Yu has indicated in his study, there are limitations to this assumption. Yet, it is a reasonable speculation that persons who shares the surname Ma may not necessarily be related while on the other hand, persons whose surnames are different may not necessarily be unrelated. Hence, the authors decided to treat a village, which usually serves a closely-knitted social unit, as a clan to study patterns in chain migration.

Additionally, Chan and Yu's Gephi visualizations are quite hard to understand. Since one of the authors' motivations is to manifest the potential of the dataset in an accessible way, Palladio, an easy-to-learn network analysis tool developed by Stanford, was chosen. The data was filtered to the county Zhongshan, and uploaded to Palladio to visualize the connections between the immigrants' origins and their destinations.

\section{Findings}

Connecting lines, node size, and proximity of nodes

Figure 3: Migration Network Visualization by Palladio, Zhongshan County 


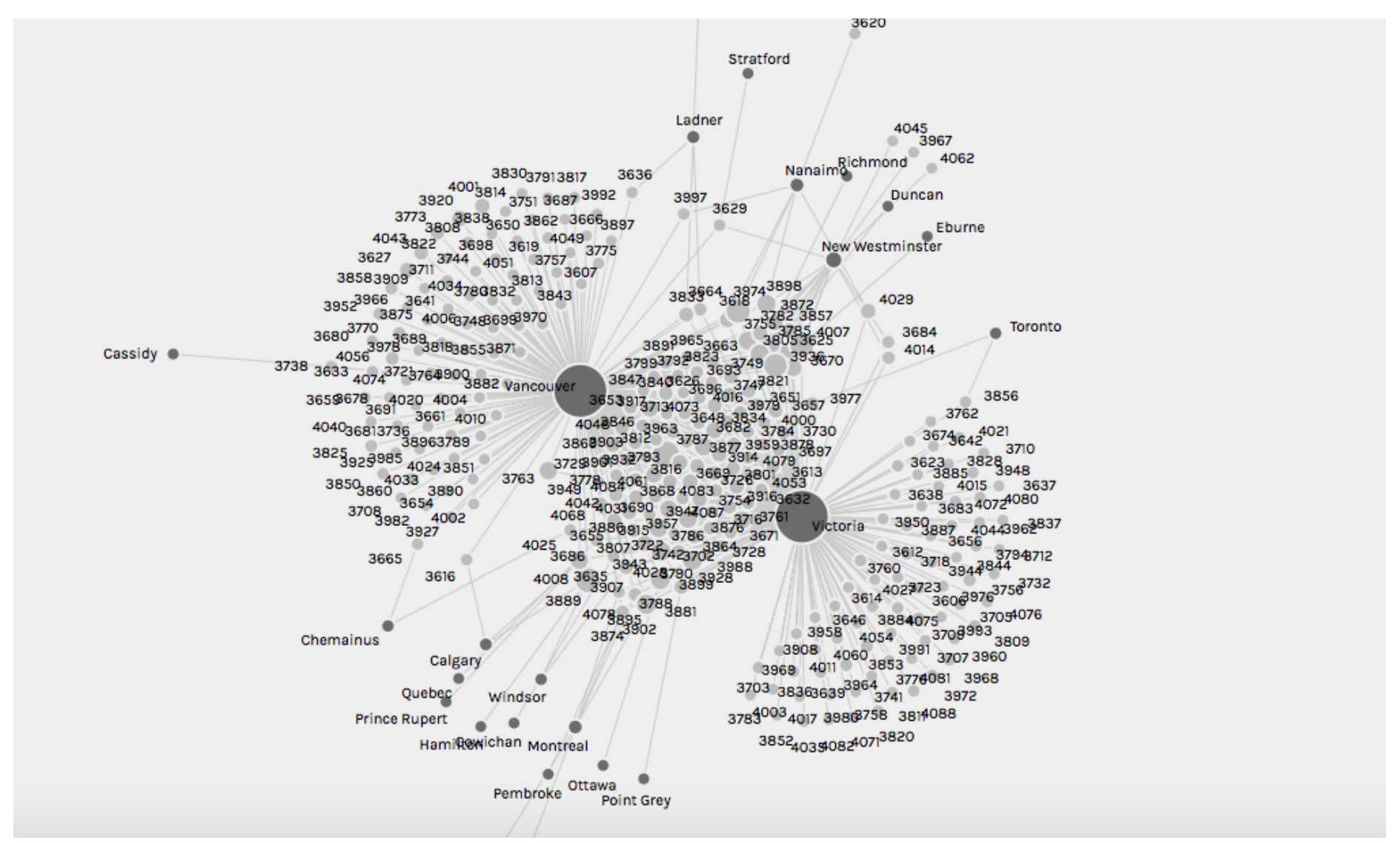

Even though the workflow of using Palladio is simple and straightforward, it takes deep thinking to truly understand the algorithm behind the scene and unlock migration patterns. This graph produced by Palladio became to be understandable after two conspicuous elements, connecting lines and node size, were investigated. Firstly, each number represents an origin village in China where immigrants were from, while the immigrants' destinations are spelled (with darker shade). Wherever a village node is connected to a destination node, it means there were immigrants originating from the village in China who chose that destination in Canada. The other feature-node size--is deceptively obvious but actually takes poking into the algorithm behind the scene in order to truly understand what it means and how it is weighted. The equation for calculating a node size can be spelled out as below: 
A node size $=\sum_{i=1}^{n} \mathrm{~A} i$

For a destination node:

$\mathrm{n}=$ how many villages the destination is connected to (how many lines a destination is connected to)

$\mathrm{A}=$ the number of immigrants each village sent

A destination node size $=$ the sum of the immigrants that each village sent which is connected to this destination.

For a village node:

$\mathrm{n}=$ how many destinations the village is connected to (how many lines a village is connected to) $\mathrm{Ai}=$ the number of immigrants each destination received

A village node size $=$ the sum of the immigrants that each destination received which is connected to this village.

In other words, a node size statistically equals the frequency of a destination or a village. It is now clear that taking a bird-view of the Palladio graph, it is a visual representation of two layers of information: which village(s) is linked to which destination(s), and the frequencies of each village and destination, all at one glance.

The third characteristic that stands out from the graph is the proximal arrangement of the nodes: nodes that are heavily connected to other nodes are placed in the middle. Vancouver and Victoria are connected to the biggest numbers of villages and so they are at the center; by contrast, the peripheral destinations are connected to fewer villages. Likewise, the villages in the middle have more connecting lines to destinations; while the two "wings" have less connecting lines to 
destinations, which means for these two big chunks of villages, most of them are only connected to one destination.

\section{Diversity of choice of destinations}

Interestingly, the two elements in Palladio visualization--a village node size and $\mathrm{n}$, how many lines a village is connected to--speak to a critical query regarding chain migration that $\mathrm{Yu}$ and Chen attempted to address: how diversified the immigrants' choices of destinations were. They found that the four major Cantonese clans in Saskatchewan all showed a high geographic distribution in choice of destination. While their findings provide a zoom-in perspective to the four clans, there are limitations to their findings for the reasons mentioned above. In order to paint a complete picture, the authors statistically studied the relationship between how many immigrants a village sent and how many destinations they ended up going.

Figure 4: The headcount and destination count, for 292 villages in Zhongshan, 1910-1949 


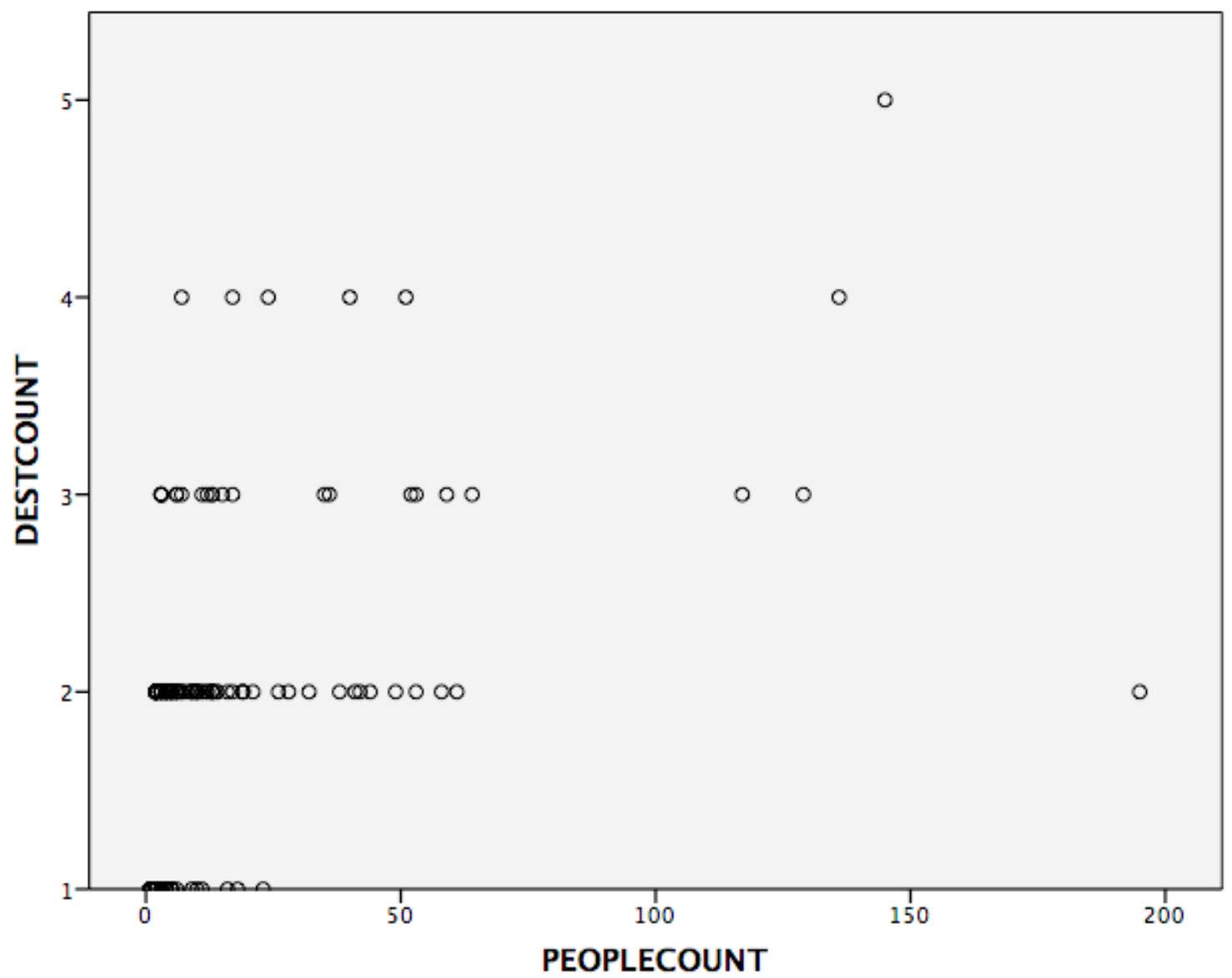

1. For Zhongshan county, most villages' choice of destinations were confined to only two, including some villages with a large number of immigrants. In other words, the immigrants originated from those villages made highly concentrated choice of destinations.

2. "Maverick" villages: For Zhongshan county, there is one village that shows extremely limited choice of destinations despite its relatively large number of immigrants.

\section{Discussions}

Based on what's discussed regarding the node size and proximity of nodes of the Palladio visualization, one of the most intriguing things that emerged is the "constellations" of village 
nodes. The proximal villages were gathered like clusters or constellations on two parameters: which destinations they were connected with, and the diversity in destinations that they were connected to. For example, the two biggest constellations of villages are connected to Vancouver and Victoria respectively. Also, the node sizes in these two constellations tend to be small to medium. Strikingly, by contrast, there is a small constellation of village nodes near the upper middle of the graph stands out from the rest. The nodes in this cluster show a couple of common characteristics: they are connected to Vancouver and Victoria but also other destinations, they are connected to relatively more destinations (higher diversity), and the nodes sizes are large (which means for these villages, they sent a relatively higher number of immigrants). Interestingly, these observations lead to a compelling research question: had these villages which showed similar migration characteristics been geographically close? This question falls to the sphere of historical GIS study; while to answer it might not be an easy task considering many old villages had disappeared or changed names, it might be a gold nugget for researchers nevertheless.

Additionally, the statistical part of the analysis--the scatter plot mapping the number of immigrants and the number of destinations for villages in two counties--revealed a different and nuanced picture than Yu and Stephanie's findings. First, the villages in Zhongshan county manifested highly limited choices of destinations, even for the villages with the relatively large number of immigrants. By contrast, the villages in Taishan county showed more diversity in the choices of destinations. Second, the number of immigrants are less correlated with the number of destinations for Zhongshan county than for Taishan county. Third, the maverick villages in Zhongshan county worth noticing. Despite the revealing of nuances in the diversity of choice of destinations, perhaps a more meaningful research question that can be asked for future studies is how the diversity of a village (clan)'s choice of destinations changed over time. The emergence of this question is illuminated in light of Yu and Chen's (2017) explanation of Myrdal's theory regarding migration patterns: 
Myrdal (1957) uses the term 'cumulative causation' to describe the snowball effect in migration patterns, whereby destinations become increasingly attractive to migrants as more of their network settles there. Thus, the best strategy for successful emigration to Canada would be to follow the example of one's relatives in choosing a destination, which a narrow interpretation of the effects of chain migration suggests. Rather than striking out on their own, newly arrived immigrants could take advantage of the connections that their relatives had already put in place, finding work and housing through families. They would have had little incentive to go elsewhere. (p.45)

Following this their conclusion is "in this scenario, a small number of origin points would connect to a relatively equal number of destinations." This, however, might be misleading or incomplete interpretation of Myrdal's theory. Another possible, and perhaps more complete, speculation would be a temporal one: to focus on how the number of destinations a village connected with changed over time. A curve can be hypothesized that the number of destinations initially increase and then after a number of years it plateaued. This hypothesis may warrant a thorough data analysis to accept or reject it.

\section{Conclusions}

The hidden patterns discovered in the study proved normalizing the county names and village/town names has allowed research work at all levels of granularity. Unfortunately, the value of normalized origins has not been systematically exploited by scholars. The findings in this study revealed much more nuanced patterns that complement or contradict previous studies, and these new findings would lead to more intriguing research questions waiting to be explored by scholars. 
As discussed, further studies are warranted to expand the understanding of early Chinese immigrants--spatially and temporally.

There might be multiple reasons for the low awareness and underuse of the data despite its substantive untapped potential. First, the normalization project is of low awareness; critical information such as how it's conducted and which counties have been normalized are missing from the webpage where the dataset inhabits. Compounding the issue is the lack of a codebook: without a meaningful codebook, it's much harder to figure out which variable is which, especially considering there are several variables regarding the immigrants' origins.

Historical data, to our amazement, has been increasingly revitalized through digitization, preservation, and provision of open access to, reaching a larger audience and allowing deeper analysis. However, there are many barriers to the computational use of data by researchers, including the challenges identified in this project. This study may represent a somewhat unique way of tackling these challenges by examining the data from two perspectives: one as if the authors are the stewards of the collection-- how to increase the usability of the dataset and make it more amenable to computational analysis; and one as a researcher--what is the potential of the data, and how computational tools can help answer and ask humanistic inquiries. Nevertheless, the authors still take the neutral stance of librarians; they didn't make any historical interpretation but simply exposed the potentials in hopes of raising awareness around the data and a history of discrimination and exclusion the data represents.

\section{References}


Hermansen, S. \& Yu, H. (2014) The Irony of Discrimination: Mapping Historical Migration Using Chinese Head Tax Data. In Fortin, M. \& Bonnell, J. (Eds). Historical GIS research in canada. Calgary: University of Calgary Press.

Yu, H. \& Chen, S. (2017). The Cantonese Pacific Migration Networks and Mobility Across Space and Time. In Wong, L., \& Yu, H.(Eds). Trans-Pacific mobilities : The Chinese and Canada. Vancouver: University of British Columbia Press.

Ward, W. P. (2013). Stature, migration and human welfare in south china, 1850-1930.Economics and Human Biology, 11(4), 488. 10.1016/j.ehb.2012.10.003 